Proceedings

\title{
OGG1 Does not Interact with NKX3.1 and AR to Repair 8-OHdG Base Damage in LNCaP Cells ${ }^{\dagger}$
}

\author{
Elif İşel 1, Bilge Debeleç Bütüner ${ }^{2}$ and Kemal Sami Korkmaz 1,* \\ 1 Department of Bioengineering, Faculty of Bioengineering Izmir, Ege University, 35040 Bornova, Turkey; \\ elifisel252@gmail.com \\ 2 Department of Pharmaceutical Biotechnology, Faculty of Pharmacy, Ege University, 35040 Bornova, Izmir, \\ Turkey; bilged79@hotmail.com \\ * Correspondence: ks_korkmaz@yahoo.com \\ + Presented at the 2nd International Cell Death Research Congress, İzmir, Turkey, 1-4 of November 2018.
}

Published: 6 December 2018

\begin{abstract}
OGG1 (8-oxo-G DNA glycosylase 1) is a BER (base excision repair) enzyme responsible for the repair of 8-oxo-G base damage related to oxidative stress through glycosylation. Determination of the interaction of DNA repair enzymes with other proteins that contributes to repairing base damages in the cell, is highly relevant in cancer treatment strategies and therapeutic targeting studies. In a previous study, it is shown that OGG1 associated with the homeobox protein NKX3.1 and androgen receptor (AR) in the 8-OHdG-repair complex in prostate cell line LNCaP, when treated with etoposide. Additionally, it is still uncertain that whether S326C, a polymorphic variant of OGG1 formed by a single amino acid change, might harbor functional loss, which might cause a deficiency in repair activity augmenting the susceptibility to (PCa). Therefore, in our study, we investigated the interaction of OGG1 and its polymorphic variant S326C with prostate specific proteins in LNCaP cells under oxidative conditions. Thus, we confirmed the previously determined interactions, however oxidative induction via menadione treatment impairs these interactions.
\end{abstract}

Keywords: OGG1; S326C; NKX3.1; AR; prostate cancer

\section{Introduction}

8-oxo-dG is a highly mutagenic lesion, yielding GC to TA transversion upon its replication by DNA polymerases [1]. 8-oxo-dG is mainly repaired by the BER mechanism. The human OGG1 gene encodes a DNA glycosylase/AP-lyase that is responsible for removing 8-oxo-dG from 8-oxo-dG: $\mathrm{dC}$ base pairs in double-stranded DNA [2]. It has been shown that the OGG1 enzyme physically interacts with a tumor suppressor protein NKX3.1, as well as with a nuclear receptor AR, which are involved in various cellular events in development and maintenance of the prostate tissue [3]. A previous study concluded that S326C had no effect on cancer risk comparison to wild type form [4]. Here, we investigated the protein-protein interactions between OGG1 enzyme and S326C variant with NKX3.1 and AR in repairing 8-oxo-dG base lesions under oxidative stress conditions.

\section{Materials and Methods}

\subsection{Cell Culture and Treatments}

LNCaP and PC3 cells were obtained from the American Type Culture Collection (ATCC, Manassas, VA, USA). The cells were propagated as recommended using RPMI 1640 medium supplemented with 10\% FBS, PC3 cells were propagated using DMEM-F12 with 5\% FBS, 
L-glutamine $(2 \mathrm{mM})$, penicillin and streptomycin $(100 \mathrm{U} / \mathrm{mL})$ at $37{ }^{\circ} \mathrm{C}$ in the presence of $5 \% \mathrm{CO}_{2}$. Menadione was purchased from Sigma-Aldrich and prepared freshly before each use. Androgen treatment was performed using R1881 $(10 \mathrm{nM})$ for $24 \mathrm{~h}$.

\subsection{Immunoprecipitation}

IP matrix (Optima F System-S. Cruz) was used and the procedure was performed as in previous publications [5].

\subsection{GST-Pull-Down}

The GST-tagged proteins were incubated with protein lysates obtained from treated LNCaP and PC3 cells. The bound proteins were precipitated with glutathione-agarose beads and analyzed by SDS-PAGE and immunoblotting with OGG1, NKX3.1 and AR antibodies.

\section{Results}

\subsection{Interaction of OGG1 with NKX3.1 and AR Has Been Confirmed by Native Immunoprecipitation}

LNCaP cells were treated with $10 \mathrm{nM}$ of R1881 for $24 \mathrm{~h}$ to activate the AR signaling pathway in LNCaP cells, and with $0.1 \mathrm{mM}$ menadione for $3 \mathrm{~h}$ to induce oxidative stress. Then, immunoprecipitation (IP) was performed to determine whether the OGG1 enzyme interacts with NKX3.1 and AR while performing the DNA repair function. Immunoprecipitation results indicated that OGG1 might be in direct physical interaction with NKX3.1 and AR (Figure 1A). Co-IP experiment was also designed to validate the IP OGG1 result, however, NKX3.1-OGG1 interaction could not be observed (Figure 1B). AR-OGG1 interaction also was negative in reciprocals (Figure 1C).

A

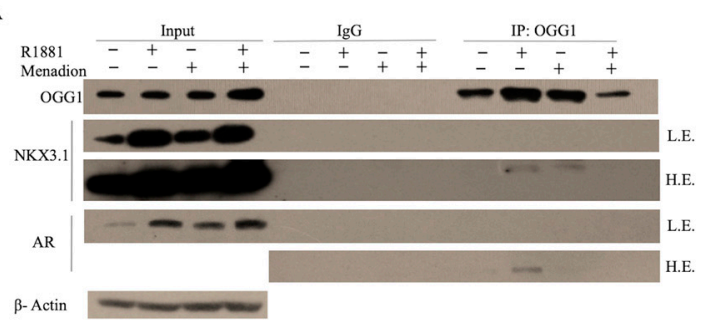

B

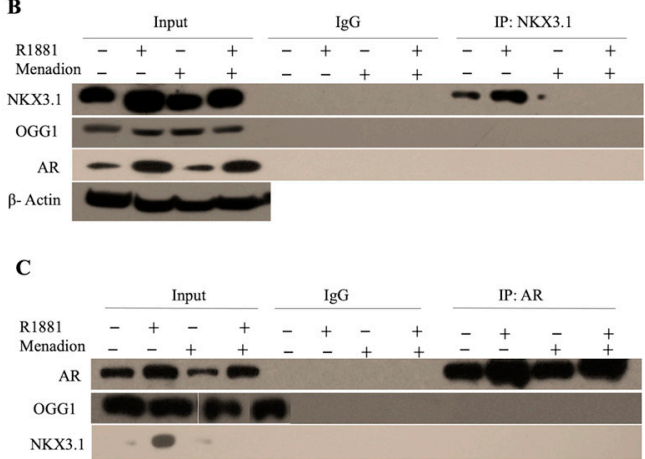

Figure 1. Physical protein-protein interaction analyses of OGG1 with NKX3.1 and AR. LNCaP cells treated with R1881 (10 nM, $24 \mathrm{~h})$ and menadione $(0.1 \mathrm{mM}, 3 \mathrm{~h})$ and then protein extracts were obtained. (A) Extracts were immunoprecipitated with a non-spesific IgG or and OGG1 antibody and then immunoblotted with NKX3.1 and AR antibody. (B) Extracts were immunoprecipitated with a non-spesific IgG or and NKX3.1 antibody and then immunoblotted with OGG1 and AR antibody. (C) Extracts were immunoprecipitated with a non-spesific IgG or and AR antibody and then immunoblotted with OGG1 and NKX3.1 antibody. L.E.: Low Exposure, H.E.: High Exposure.

\subsection{Interaction of OGG1 and S326C Its Polymorphic Variant with NKX3.1 and AR Has not Been Observed by} GST-Pull Down Assay

To investigate the effect of S326C variation on interactions of OGG1 with AR and NKX3.1, we tested the binding rates of both wild-type $(\mathrm{wt})$ and polymorphic variant proteins by using GST pull-down precipitation method. Using GST-OGG1-wt and GST-OGG1 (S326C) constructs, proteins were expressed in E. coli and isolated from lysate. Since, GST and OGG1 are 25 and $39 \mathrm{kDa}$ respectively, GST-OGG1 fusion protein was expected to be $65 \mathrm{kDa}$, which was observed as it is (Figure 2A). Thereafter, if the glutathione agarose beads were then used to precipitate these protein 
lysates (Figure 2B) and checked by OGG1 antibody labeling (Figure 2C), it was positive whereas AR was not observed in samples 6 and 8. Therefore, we concluded that OGG1-AR interaction was not positive. In the NKX3.1 antibody labeling, there was a significant band in samples 6 and 8, however, non-specific NKX3.1 bands in negative controls were also observed so that the bands did not indicate the specific interactions (Figure 2D). Therefore the conclusion was also negative for both protein interactions tested.

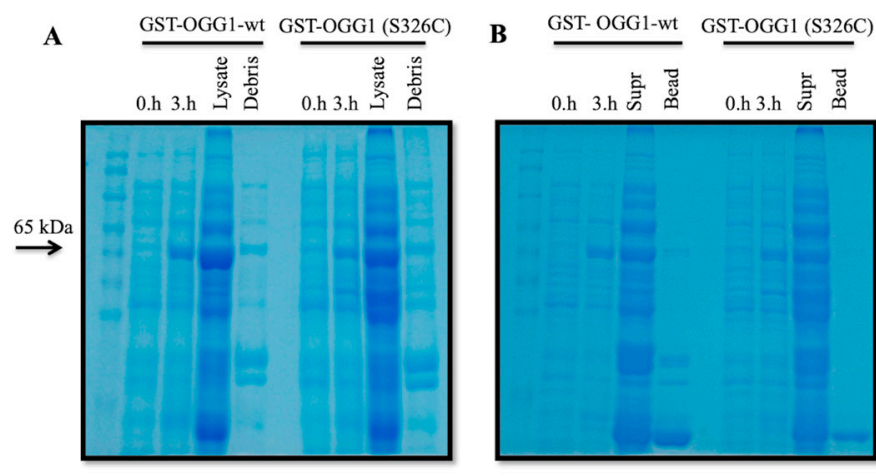

C

$\underline{\text { GST-OGG1 Sup-1 }} \underline{\text { Bead-2 }} \underline{\text { Bead-1 }}$ Sup-2

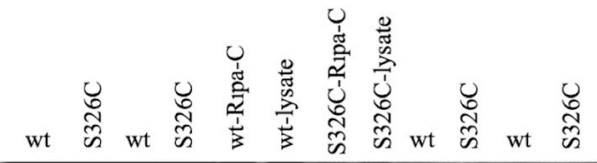

OGG1

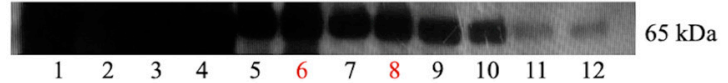

D

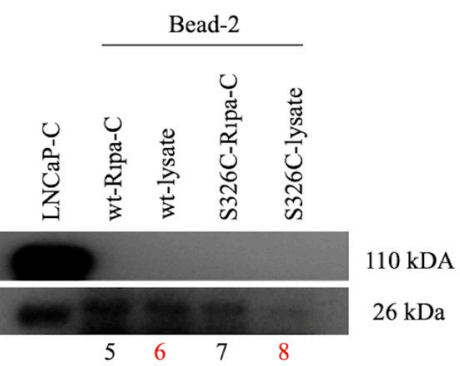

E
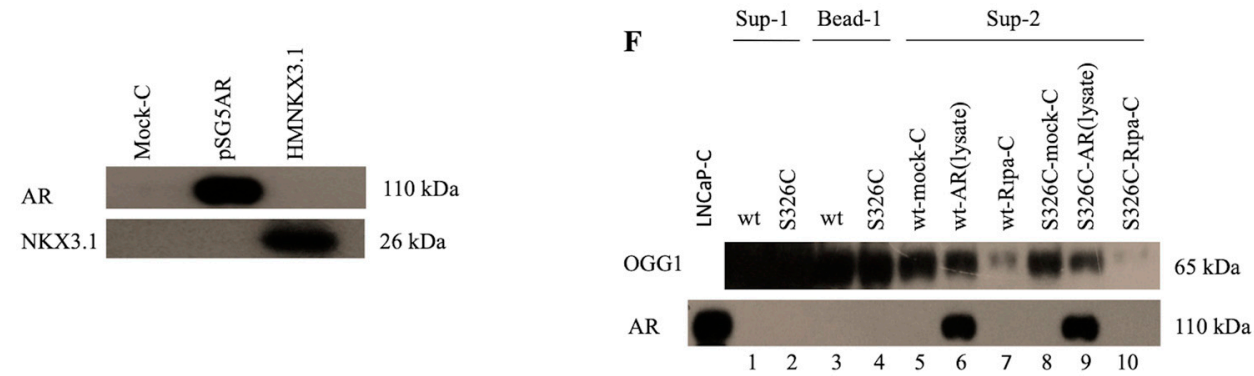
G

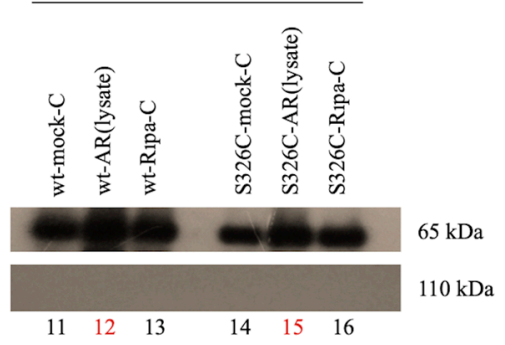

H

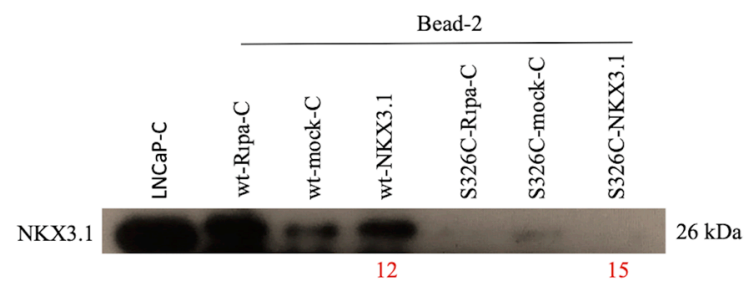

Figure 2. A. Control of GST-OGG1 (wt) and GST-OGG1 (S326C) proteins from E. coli transformants by comassie blue staining and western blot images showing protein-protein interaction analysis of GST-OGG1 (wt)/(S326C) proteins with NKX3.1 and AR. (A) After the in crease of GST-OGG1 (wt)/S326C) protein expression with $1 \mathrm{mM} \mathrm{IPTG}$ induction, bacterial protein isolation was performed and checked with comassie blue staining. (B) Control of glutathione agarose beads. (C) GST-OGG1 (wt) and GST-OGG1 (S326C) that precipitated with glutathione agarose beads were treated with LNCaP protein lysates. To find out if OGG1 is bound to beads and pulled down, first labeled with OGG1 antibody. (D) To examine protein-protein interaction, membrane immunoblotted with AR and NKX3.1 antibodies. 6 and 8 are examples that show protein-protein interaction. (E) Ectopic expression of AR and NKX3.1 in PC3 cells is shown. GST-OGG1 (wt) and GST-OGG1 (S326C) proteins were treated with PC3 cell lysates that expresses NKX3.1 and AR ectopically. (F) To find out if OGG1 is bound to beads and pulled down, first labeled with OGG1 antibody. The AR protein was checked by AR antibody labeling. (G) 12 and 15 are examples that show OGG1-AR protein-protein interaction. (H) 12 and 15 are examples that show OGG1-NKX3.1 protein-protein interaction.

\section{Discussion}

Bowen et al. showed that the OGG1 enzyme interacts with AR and NKX3.1, which was shown using the native IP protocol [3], upon etoposide treatment in LNCaP cells. In our study, we first observed the interaction in native IP-OGG1 in LNCaP cells. Thereafter, analyzed the PC3 cells that ectopically expressed NKX3.1 and AR, in addition to GST-Pull down in LNCaP cells. Overall, we could not show specific interaction of OGG1wt and mutant OGG1S326C with NKX3.1 and AR. As we used menadione to induce oxidative DNA damage in LNCaP cells, there might be a different mechanism, which is activated to trigger DNA damage repair due to the substances used. Etoposide causes DNA damage via topoisomerase-II inhibition. However, menadion creates oxidation of guanine to make base damage. Therefore, a direct intervention on topoisomerase enzyme given to the cell by etoposide may cause NKX3.1 and AR stabilization and increase the protein-protein linkage with the OGG1 enzyme from the site to perform DNA repair. Nevertheless the oxidative stress created by menadione may not pose a serious danger in comparison to the damage caused by etoposide. Thus, it may not enough impose the interaction of OGG1 with NKX3.1 and AR as it is required for a certain threshold. It should also be noted that, many proteins could interact with the OGG1 protein via short or long-term interactions in the repair of 8-oxo-dG lesions in different cellular and damage conditions. So that, in the case of each cellular stress, OGG1 protein does not continuously interact with NKX3.1 and AR to contribute to repair of DNA damage in LNCaP cells especially under oxidative conditions, which require BER activation.

Acknowledgments: This research was supported from Tübitak grant no: $113 S 044$ to KSK and BDB. Additoanally thanks to Radicella for GST fused OGG1 vectors.

\section{References}

1. Boiteux, S.; Radicella, J.P. The Human OGG1 Gene: Structure, Functions, and Its Implication in the Process of Carcinogenesis. Arch. Biochem. Biophys. 2000, 377, 1-8. 
2. Luna, L.; Rolseth, V.; Hilgrestrand, G.A.; Otteriei, M.; Dantzer, F.; Bjøra, M.; Seeberg, E. Dynamic relocalization of hOGG1 during the cell cycle is disrupted in cells harbouring the hOGG1-Cys326 polymorphic variant. Nucleic Acids Res. 2005, 33, 1813-1824.

3. Bowen, C.; Zheng, T.; Gelmann, E.P. NKX3.1 Suppresses TMPRSS2-ERG Gene Rearrangement and Mediates Repair of Androgen Receptor Induced DNA Damage. Can. Res. 2015, 75, 2686-2698.

4. Weiss, J.M.; Goode, E.L.; Ladiges, W.C.; Ulrich, C.M. Polymorphic Variation in hOGG1 and Risk of Cancer: A Review of the Functional and Epidemiologic Literature. Mol. Carcinog. 2005, 42, 127-141.

5. Debelec-Butuner, B.; Alapinar, C.; Varişli, L.; Erbaykent-Tepedelen, B.; Muhammad Hamid, S.; Gonen-Korkmaz, C.; Korkmaz, K. Inflammation-mediated abrogation of androgen signaling: An in vitro model of prostate cell inflammation. Mol. Carcinog. 2014, 53, 85-97.

(c) 2018 by the authors. Licensee MDPI, Basel, Switzerland. This article is an open access article distributed under the terms and conditions of the Creative Commons Attribution (CC BY) license (http://creativecommons.org/licenses/by/4.0/). 